\title{
Stochastic parcel tracking in an Euler-Lagrange compartment model for fast simulation of fermentation processes
}

\author{
Cees Haringa ${ }^{1}$, Wenjun Tang ${ }^{2}$, and Henk Noorman ${ }^{3}$ \\ ${ }^{1}$ Technische Universiteit Delft Faculteit Technische Natuurwetenschappen \\ ${ }^{2}$ Royal DSM \\ ${ }^{3} \mathrm{DSM}$
}

September 27, 2021

\begin{abstract}
Compartment modeling (CM) is a well-known approach for computationally affordable, spatially-resolved hydrodynamic modeling of unit operations. Recent implementations use flow profiles based on CFD simulations, and several authors included microbial kinetics to simulate gradients in bioreactors. However, these studies relied on black-box kinetics, that do not account for intra-cellular changes and cell population dynamics in response to heterogeneous environments. In this paper, we report the implementation of a Lagrangian reaction model, where the microbial phase is tracked as a set of biomass-parcels, each linked with an intra-cellular composition vector and a structured reaction model describing their intra-cellular response to extracellular variations. A stochastic parcel tracking approach is adopted, in contrast to the resolved trajectories used in prior CFD implementations. A penicillin production process is used as a case-study. We show good performance of the model compared to full CFD simulations, both regarding the extra-cellular gradients and intra-cellular pool response, provided the mixing time in the CM matches the full CFD simulation; taking into account that the mixing time is sensitive to the number of compartments. The sensitivity of the model output towards some of the inputs is explored. The coarsest representative CM requires a few minutes to solve 80 hours of flow time, compared to approx. 2 weeks for a full Euler-Lagrange CFD simulation of the same case. This alleviates one of the major bottlenecks for the application of such CFD simulations towards analysis and optimization of industrial fermentation processes.
\end{abstract}

\section{Hosted file}

stochastic_review_version.pdf available at https://authorea.com/users/438133/articles/539396stochastic-parcel-tracking-in-an-euler-lagrange-compartment-model-for-fast-simulationof-fermentation-processes 